NOTA CIENTÍFICA

\title{
Evaluación de sustratos para el establecimiento en laboratorio de plántulas de Puya raimondii Harms (Bromeliaceae)
}

\section{Evaluation of substrates for seedlings establishment in laboratory of Puya raimondii Harms (Bromeliaceae)}

\author{
Giovana Vadillo* y Mery Suni
}

\begin{abstract}
* Laboratorio de Fisiología Vegetal. Instituto de Investigaciones de Ciencias Biológicas Antonio Raimondi. Facultad de Ciencias Biológicas. Universidad Nacional Mayor de San Marcos. Lima. Perú.

E-mail Giovana Vadillo: gpvadillo@hotmail.com
\end{abstract}

Presentado: $\quad 02 / 02 / 2006$ Aceptado: $\quad 28 / 08 / 2006$

\begin{abstract}
Resumen
Puya raimondii Harms (Bromeliaceae) es una planta monocárpica que puede producir entre 6 y 12 millones de semillas, sin embargo es muy raro encontrar plántulas en su hábitat natural, lo que significaría la existencia de factores limitantes en el proceso de establecimiento. En el presente trabajo muestra los resultados de experimentos para determinar el sustrato más adecuado para el establecimiento de $P$. raimondii bajo condiciones de laboratorio. Se comparó la sobrevivencia y vigor de plántulas de $P$. raimondii en los substratos de turba, musgo, turba-tierra y musgo-tierra. El sustrato turba presentó la mayor supervivencia, mostrando que es el más adecuado para el establecimiento de las plántulas, porque mantiene una humedad adecuada y $\mathrm{pH}$ estable lo que permitiría que las plántulas presenten un mayor vigor; los sustratos con muy poca (caso turba-tierra) o demasiada (caso musgo) retención de humedad afectan negativamente su vigor y desarrollo.
\end{abstract}

Palabras clave: Puya raimondii, Bromeliaceae, plántulas, establecimiento de plántulas.

\section{Abstract}

Puya raimondii Harms (Bromeliaceae) is a monocarpic plant, that can produce about 6 to 12 million seeds, nevertheless is very rare to find seedlings in their natural habitat, indicating difficulties for its establishment. In this work, the conditions for establishment of $P$. raimondii under laboratory conditions were determined carrying out experiments to determine the better substrate. It was compared the survival and vigour of $P$. raimondii seedling that were transplanted in peat, moss, peat-soil and moss-soil substrates. The peat substrate showed high survival and was better for establishment of seedlings because it maintains steady and suitable humidity and $\mathrm{pH}$ conditions, this allowed that seedlings presents a good vigour. Substrates with very little (case peat-earth) or too much (case moss) humidity retention had a negative effect over vigour and development.

Keywords: Puya raimondii, Bromeliaceae, seedling establishment, seedling.
Puya raimondii Harms es una especie de la subfamila Pitcairnioideae, familia Bromeliaceae distribuida en la Puna de los Andes de Perú y Bolivia. Se le encuentra preferentemente en las laderas de los cerros con exposición al noreste y noroeste, donde hay mayor radiación solar; preferentemente en terrenos rocosos o pedregosos con pendientes moderadas a muy fuertes (Villiger, 1981; Venero, 1984; Rivera, 1985).

Puya raimondii dispersa entre 6 a 12 millones de semillas (Rivera, 1985) durante el mes de julio en el siguiente año de la floración (Suni et al., 2002), antes que se inicien las lluvias. A pesar de que buena parte de sus semillas pueden tener alto poder germinativo; los daños ocasionados por las aves, hongos y larvas de polilla durante la fructificación disminuyen el número de semillas viables y vigorosas disminuyendo su capacidad de dispersarse. Adicionalmente, los requerimientos para su germinación (luz, humedad adecuada y temperatura) limitan los microhábitats que pueden colonizar. A esto se agrega el efecto negativo del hombre, con actividades como el pastoreo y la expansión de las zonas agrícolas, las cuales disminuyen aun más la posibilidad de su establecimiento (Vadillo et al., 2004). Como consecuencia de lo mencionado, es muy raro encontrar plántulas (menores a $5 \mathrm{~cm}$ ) en su hábitat natural.
En referencia a los factores que influyen el crecimiento de la plántula se tieneinformación general para las bromelias. Smith y D owns (1973) mencionan que uno de los factores es la disponibilidad del agua. Los sustratos generalmente usados son las mezclas de musgo, turba y grava (Smith \& D owns, 1973).

En el presente estudio se evaluaron cuatro diferentes sustratos: musgo (Sphagnum sp.), turba (D istichia muscoides), musgo-tierra (1:1) y turba-tierra (1:1); cada tipo de sustrato con seis repeticiones (bandejas). Se transplantó 24 plántulas que tenían dos hojas (dos meses de germinada, aproximadamente) a cada bandeja de 10 x 7,5 cm con $2 \mathrm{~cm}$ de profundidad. Las bandejas fueron distribuidas al azar y colocadas en condiciones de laboratorio (temperaturas entre 20 y $30^{\circ} \mathrm{C}$, y humedades relativas entre $100 \%$ y $60 \%$, para noche y día, respectivamente). D espués de 36 días de instalado el experimento se redujo a 12 el número de las plántulas (raleo).

Los sustratos fueron previamente tamizados para homogenizar a partículas menores de $2 \mathrm{~mm}$. Al inicio del experimento se evaluó el pH, conductividad eléctrica (CE), densidad, porosidad y al final de la prueba el pH y CE . Las bandejas fueron regadas dos a tres veces por semana solo con agua destilada ( $\mathrm{pH}$ 
Tabla 1 Evaluación de algunas características de los diferentes sustratos utilizados en el establecimiento de plántulas de Puya raimondii.

\begin{tabular}{|c|c|c|c|c|c|c|c|c|}
\hline \multirow[b]{2}{*}{ Sustratos } & \multicolumn{2}{|c|}{ pH } & \multicolumn{2}{|c|}{$\overline{C E}$} & \multirow{2}{*}{$\begin{array}{c}\text { Porosidad } \\
\%\end{array}$} & \multirow{2}{*}{$\begin{array}{l}\text { Capacidad } \\
\text { de Campo } \\
\%\end{array}$} & \multirow{2}{*}{$\begin{array}{l}\text { humedad } \\
\text { retenida \% } \\
5 \mathrm{ddr}\end{array}$} & \multirow{2}{*}{$\begin{array}{l}\text { Densidad } \\
\text { A parente } \\
(\mathrm{g} / \mathrm{ml})\end{array}$} \\
\hline & Inicio & Final & Inicio & Final & & & & \\
\hline musgo & 4,6 & 7,2 & 98,0 & 49,0 & 88,9 & 98,0 & 49,0 & 0,05 \\
\hline M usgo-tierra & 4,8 & 5,4 & 72,3 & 36,1 & 41,2 & 72,3 & 36,1 & 0,73 \\
\hline turba & 6,4 & 7,1 & 96,2 & 48,1 & 87,4 & 96,2 & 48,1 & 0,10 \\
\hline turba-tierra & 6,2 & 7,3 & 75,5 & 37,7 & 34,7 & 75,5 & 37,7 & 0,65 \\
\hline HDS & & & 4,19 & 1,77 & 2,08 & 4,19 & 1,77 & \\
\hline
\end{tabular}

Separación de medias por el factor HDS de Tukey $(p=0,05)$; entre sustratos. CE: conductividad eléctrica. ddr: Días después del riego

7,1; CE 0,0). Posteriormente se regó una vez por semana con solución nutritiva (N: 205 ppm, P: 55 ppm, K: 208 ppm, Ca: 118 ppm, Mg: 124 ppm, S: 53 ppm, FeDD HA ymicronutrientes) amedia concentración.

Se evaluó el porcentaje de sobrevivenciay el vigor dela plántula mediante el número de hojas totales, número de Hojas verdesy cobertura (área superficial). Los datos obtenidos por cada tratamiento y cada tiempo fueron promediados para el Análisis de Varianza, la comparación y el análisis de medias con la prueba de Tukey HD S con un nivel de confianza del 95\% (Tabla 2).

Las características iniciales y finales de los diferentes sustratos utilizados se presentan en la Tabla 1; al inicio el sustrato musgo (Sphagnum sp.) presentaban pH más ácido y más baja CE que la turba (D istichia muscoides); pero al finalizar el experimento el pH se incrementó en todos los sustratos siendo mayor en musgo y turba-tierra mientras la CE se mantuvo constante en el sustrato turba.

En cuanto al porcentaje de sobrevivencia (Tabla 2) se obtuvo diferencias significativas $(P<0,05)$ entre los sustratos evaluados a los $9 \mathrm{D}$ dt (Días después del transplante) incrementándose esta diferencia $(\mathrm{p}<0,01)$ a los $18 \mathrm{D}$ dt, encontrándose mayor mortandad en el sustrato de turba-tierra, obteniéndose los mejores resultados en los sustratos de turba y musgo-tierra; pero a partir delos $33 \mathrm{D}$ dt no se encontró diferencias significativas.

Hasta los 32 D dt se obtuvo un incremento homogéneo del número de hojas totales. En las siguientes fechas comenzó a aparecer diferencias significativas ( $p<0,01)$ las que se van acentuando con el tiempo hasta los $56 \mathrm{D}$ dt. Las plántulas del sustrato

Tabla 2. Variación de los diferentes parámetros evaluados por sustrato en el tiempo. Análisis de variancia ANOVA y separación de medias por la prueba de Tukey.

\begin{tabular}{|c|c|c|c|c|c|c|}
\hline $\begin{array}{l}\text { Fecha eval. } \\
\text { D draleo } \\
\text { D dt }\end{array}$ & $\begin{array}{c}01 / 08 / 01 \\
9\end{array}$ & $\begin{array}{c}10 / 08 / 01 \\
18\end{array}$ & $\begin{array}{c}25 / 08 / 01 \\
33\end{array}$ & $\begin{array}{c}01 / 09 / 01 \\
4 \\
40\end{array}$ & $\begin{array}{c}15 / 09 / 01 \\
18 \\
44\end{array}$ & $\begin{array}{c}28 / 09 / 01 \\
31 \\
57\end{array}$ \\
\hline & \multicolumn{6}{|c|}{ Porcentaje de sobrevivencia (\%) } \\
\hline turba & $100 \mathbf{a}$ & $97,9 \mathbf{a}$ & 93,8 & 100 & 98,6 & 97,2 \\
\hline turba-tierra & 92,4 b & 91,7 b & 91,7 & 100 & 100 & 100 \\
\hline musgo & $99,3 \mathbf{a b}$ & $96,5 \mathbf{a b}$ & 88,9 & 100 & 100 & 100 \\
\hline musgo-tierra & $100 \mathbf{a}$ & 100 a & 95,8 & 100 & 100 & 100 \\
\hline \multirow[t]{2}{*}{ HDS } & 7,05 & 6,09 & 15,15 & 0,0 & 1,54 & 3,48 \\
\hline & \multicolumn{6}{|c|}{$\mathrm{N}$ úmero de $\mathrm{Hojas}$ Totales } \\
\hline D dt & 8 & 17 & 32 & 39 & 43 & 56 \\
\hline turba & 2 & 3 & 4.1 & 6,3 a & $8,0 \mathbf{a}$ & 8,5 a \\
\hline turba-tierra & 2 & 3 & 4 & 4,4 b & $5,3 \mathrm{c}$ & $6,3 \mathrm{c}$ \\
\hline musgo & 2 & 3 & 4 & 6,1 a & 6,8 b & 7,2 b \\
\hline musgo-tierra & 2 & 3 & 4 & 6,4 a & 8,0 a & $7,9 \mathbf{a b}$ \\
\hline \multirow[t]{2}{*}{ HDS } & 0,0 & 0,0 & 0,18 & 0,76 & 0,72 & 0,91 \\
\hline & \multicolumn{6}{|c|}{$\mathrm{N}$ úmero de $\mathrm{Hoj}$ as $\mathrm{V}$ erdes } \\
\hline D dt & 8 & 17 & 32 & 39 & 43 & 56 \\
\hline turba & 2 & 3 & 4,1 & $6,3 \mathbf{a}$ & $7,7 \mathbf{a}$ & 5,7 \\
\hline turba-tierra & 2 & 3 & 4 & 4,4 b & $4,5 \mathrm{c}$ & 4 \\
\hline musgo & 2 & 3 & 4 & 6,1 a & $6,0 \mathbf{b}$ & 5 \\
\hline \multirow{3}{*}{$\begin{array}{l}\text { musgo-tierra } \\
\text { HDS }\end{array}$} & 2 & 3 & 4 & 6,4 a & 7,0 a & 6,7 \\
\hline & 0,0 & 0,0 & 0,18 & 0,76 & 0,93 & 2,90 \\
\hline & \multicolumn{6}{|c|}{ Cobertura $\left(\mathrm{mm}^{2}\right)$} \\
\hline D dt & 8 & 17 & 32 & 39 & 43 & 56 \\
\hline turba & & 5,2 & 9,9 a & $12,7 \mathbf{a}$ & 33,8 a & 50,5 a \\
\hline turba-tierra & & 4,5 & $5,5 \mathbf{b}$ & $6,8 \mathbf{b}$ & $8,9 \mathrm{c}$ & $12,0 \mathbf{d}$ \\
\hline musgo & & 5,3 & 9,8 a & 13,5 a & $21,3 \mathbf{b}$ & 29,2 c \\
\hline musgo-tierra & & 5,5 & 8,6 a & 14,4 a & 30,8 a & 39,8 b \\
\hline HDS & & 1,55 & 3,18 & 3,97 & 8,11 & 10,47 \\
\hline
\end{tabular}

Separación de medias por el factor HDS de Tukey $(\mathrm{p}=0,05)$. Valores con la misma letra son estadísticamente iguales. Ddt: días después del transplante, Ddraleo: días después del raleo 
turba tuvieron los valores mayores para el incremento de hojas seguidas de las de musgo-tierra y musgo, y finalmente de turbatierra.

El número de hojas verdes por planta fue similar hasta los 32 días para los diferentes sustratos. En las siguientes fechas de evaluación se apreciaron diferencias, siendo los sustratos de turbay musgo-tierra los que presentaron mayor número de hojas verdes hasta los $43 \mathrm{Ddt}$, sin embargo, a los $56 \mathrm{Ddt}$ ya no se encuentra diferencias significativas sino una tendencia donde el sustrato musgo-tierra aparece con mayores valores.

En el caso de la cobertura de las plántulas se encontró diferencias significativas $(p<0,01)$ entre los sustratos a partir de los 32 D dt las cuales se acentúa con el tiempo llegándose a diferenciar cada sustrato individualmente. El sustrato turba presentó los mayores valores de cobertura.

Se puedo notar que para todos los parámetros evaluados el sustrato turba es el que tiene los mejores resultados seguido de musgo-tierra.

\section{Discusión}

La sobrevivencia de las plántulas, evaluados durante dos meses, fue alta (>90\%) para todos los sustratos, aunque el vigor de las plántulas varió. Las primeras diferencias significativas se encontraron a los 17 días después del transplante (D dt) para la cobertura y a los $39 \mathrm{D}$ dt en el número de hojas totales; en ambos casos las plántulas del sustrato turba-tierra presentaron los menores valores, acentuándose esta diferencia, a medida que transcurría el tiempo hasta el final (57 Ddt), donde las plántulas del sustrato turba presentaron los mejores valores para los parámetros evaluados (Tabla 2).

Las diferencias encontradas en el vigor de las plántulas, pueden explicarse por las diferencias en retención de la humedad de los sustratos, donde la escasa (caso turba-tierra) o excesiva (caso musgo) retención de humedad por el sustrato, influenciaron negativamente en el vigor de las plántulas. La retención de humedad de cada sustrato esta relacionado con su porosidad, su capacidad de campo y su densidad aparente (Tabla 1). Además el $\mathrm{pH}$, durante el experimento, varió poco $(0,7$ y 0,6$)$ en los sustratos de turba y musgo-tierra mientras que en los sustratos musgo y turba-tierrala variación fuemayor (2,6 y 1,1 respectivamente). La turba mostró ser un sustrato con características adecuadas para el establecimiento de plántulas en invernadero. Estos resultados están en concordancia con lo que menciona Smith y D owns (1973) quienes mencionan que los factores que influyen en el crecimiento de las bromelias son la disponibilidad del agua y aireación.
El éxito del establecimiento de las plántulas deP Pya raimondii en su hábitat parece depender de las zonas en que ocurra la germinación; aquellas plántulas que emergen en las zonas rocosas, pedregosas o entre pajonales o musgos tienen mayor probabilidad de establecerse, debido a que estos microhábitats presentan suelos ricos en materia orgánica y la humedad, aireación y radiación adecuadas. Así mismo, les brindan protección a las fuertes oscilaciones de temperatura hasta que desarrollen las adaptaciones y el tamaño adecuado que les permitan soportar las condiciones en que viven. Esto explica la mayor frecuencia de esta especie en lugares rocosos y pedregosos. Sin embargo como señalara Raimondi (1984), el alto número de semillas (millones) que forma esta especie seria su mejor estrategia para asegurar el establecimiento de sus plántulas en el hábitat y la permitir la continuidad de la especie.

\section{Literatura citada}

Besnier, R. 1989. Semillas Biología y Tecnología. Ed. Madrid España. 524 pp.

Raimondi, A. 1874. El Perú. Tomo 1, parte preliminar. Imprenta del Estado, Calle de la Rifa. Num. 58 Págs. 293-297

Rivera, C.A. 1985. Puya raimondii Harms. Boletín de Lima 38: 8591

Smith, L. B. \& L. J. Downs. 1973. Flora neotrópica. Monograph $\mathrm{N}^{\circ} 14$, Part 3. Organization for flora neotropica by the New York Botanical Garden. USA. 189-190

Suni, M., A. Cano, J. Fernández \& G. Vadillo. 2002. Avances en el estudio de la floración de Puya raimondii Harms (Bromeliaceae). Resumen del XI Reunión Científica ICBAR. Pág. 57

Vadillo, G.; M. Suni y A. Cano. 2004. Viabilidad y germinación de semillas de Puya raimondii Harm (Bromeliaceae). Rev. peru. biol. 11(1):71-78

Venero, J.L. 1984. El rodal de Puya raimondii en Lares Calca. Boletín de lima 31:65-69

Villiger, F. 1981. Rodales de Puya raimondii y su protección. Boletín de Lima 10:84-91 


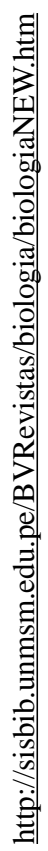

142 